\title{
A NOTE ON METRIZATION OF MOORE SPACES
}

\section{REGINALD TRAYLOR}

J. N. Younglove recently has proved [3] that a normal, complete Moore space is metrizable, provided that the boundary of each domain is screenable. It is established in the first theorem of this paper that the same conclusion follows without the requirement of completeness on the space. The second theorem establishes a relation between separability and pointwise paracompactness in complete Moore spaces.

The statement that the space $S$ is a Moore space means there is a sequence of collections of regions in $S$ satisfying Axiom 0 and Axiom $1_{3}$ of [2]. A Moore space is complete if and only if there is a sequence of collections of regions in the space satisfying all of Axiom 1 of [2].

The statement that the point set $M$ is screenable means that if $G$ is an open covering of the space $S$, there exists a sequence $H_{1}, H_{2}$, $\mathrm{H}_{3}, \cdots$ of collections of mutually exclusive open sets such that $\sum H_{i}$ is a refinement of $G$ which covers $M$.

The statement that the point set $M$ is pointwise paracompact means that if $G$ is an open covering of the space $S$, there is an open refinement $H$ of $G$ such that $H$ covers $M$ and no point of $S$ belongs to infinitely many elements of $H$.

Theorem 1. A normal Moore space is metrizable if and only if the boundary of each domain is screenable. ${ }^{1}$

Proof. Suppose $S$ is a normal Moore space and $H$ is a covering of $S$. Denote by $H^{\prime}$ a collection of mutually exclusive domains such that $H^{\prime *}$ is dense in $S$ and $H^{\prime}$ refines $H$. But $H^{* *}$ is a domain so $S-H^{\prime *}$ is screenable. Then there exists a sequence $H_{1}, H_{2}, H_{3}, \cdots$ such that each $H_{i}$ is a collection of mutually exclusive domains, each of which is a subset of some element of $H$, and $\sum H_{i}$ covers $S-H^{\prime *}$. In this case, $H^{\prime}, H_{1}, H_{2}, H_{3}, \cdots$ screens $S$ with respect to $H$ and thus, $S$ is a screenable space. Bing has proved [1] that each normal, screenable space is metrizable.

Since Bing has proved also [1] that each metrizable Moore space is screenable, it follows that the boundary of each domain of a metrizable Moore space is screenable.

Received by the editors April 13, 1962 and, in revised form, June 27, 1962.

I It is my understanding that E. E. Grace has an independent but similar proof of this theorem. 
TheOREM 2. A separable, complete Moore space is metrizable if and only if the boundary of each domain is pointwise paracompact.

Proof. Suppose $S$ is a separable, complete Moore space. Younglove has proved [4] that there exists a metrizable subspace $S^{\prime}$ which is dense in $S$. The point set $h$ is a domain in $S^{\prime}$ if and only if there exists a domain $g$ in $S$ such that $h$ is $g \cdot S^{\prime}$.

Suppose $G$ is an open covering of $S$ and $H$ is the collection to which $h$ belongs if and only if there is an element $g$ of $G$ such that $h$ is $g \cdot S^{\prime}$. In $S^{\prime}, H$ is an open covering of $S^{\prime}$. A theorem proved by Bing [1] establishes that there is a sequence $H_{1}^{\prime}, H_{2}^{\prime}, H_{3}^{\prime}, \cdots$ of discrete (in $S^{\prime}$ ) collections of open sets such that the sum of the collections of the sequence is a refinement of $H$ which covers $S^{\prime}$. For each element $h^{\prime}$ of each $H_{i}^{\prime}$, denote by $h$ some domain in $S$ such that $h \cdot S^{\prime}$ is $h^{\prime}$. If $H_{i}$ is the collection to which the domain $d$ belongs if and only if there is a domain $h^{\prime}$ of $H_{i}^{\prime}$ such that $d$ is $h$, then $H_{i}$ is a collection of mutually exclusive domains. For suppose each of $h$ and $h_{1}$ belong to $H_{i}$ and $h$ intersects $h_{1}$. Then $h \cdot h_{1}$ is open and $h \cdot S^{\prime}$ intersects $h_{1} \cdot S^{\prime}$. This means that $H_{i}^{\prime}$ is not a discrete collection of domains in $S^{\prime}$ and a contradiction is reached.

Since $S$ is separable, each $H_{i}$ is a countable collection. Since $S-\sum H_{i}^{*}$ is pointwise paracompact, there is a refinement $G^{\prime}$ of $G$ covering $S-\sum H_{i}^{*}$ such that no point of $S$ belongs to infinitely many of the elements of $G^{\prime}$. But $S$ is separable, so $G^{\prime}$ must be countable.

It is clear that if $H^{\prime}$ is the collection to which the domain $d$ belongs if and only if $d$ is a domain of $G^{\prime}$ or of some $H_{i}$, then $H^{\prime}$ is countable. Thus, $S$ is completely separable, and therefore metrizable.

If the space is metrizable, it is paracompact. In this case, it is obvious that the boundary of each domain is pointwise paracompact.

\section{REFERENCES}

1. R. H. Bing, Metrization of topological spaces, Canad. J. Math. 3 (1951), 175-186.

2. R. L. Moore, Foundations of point set theory, Amer. Math. Soc. Colloq. Publ. Vol. 13, Amer. Math. Soc., Providence, R. I., 1932.

3. J. N. Younglove, $A$ theorem on metrization of Moore spaces, Proc. Amer. Math. Soc. 12 (1961), 592-593.

4. - Concerning dense metric subspaces of certain nonmetric spaces, Fund. Math. 48 (1959), 15-25.

AUBURN University 